\title{
Escala de Compasión (ECOM) para población mexicana ${ }^{1}$
}

\section{Compassion Scale (ECOM) for Mexican population}

\author{
Alejandro López Tello y Ana Beatriz Moreno Coutiño ${ }^{2}$ \\ Citación: López T., A y Moreno C., A.B. (2019). Escala de Compasión (ECOM) para \\ población mexicana. Psicología y Salud, 29(1), 25-32.
}

\section{RESUMEN}

\begin{abstract}
El constructo compasión utilizado en el presente estudio se definió como el profundo deseo de aliviar el sufrimiento propio y el de los demás. En esta investigación se describe el proceso de construcción y evaluación de las propiedades psicométricas de una escala de compasión para población mexicana. La muestra estuvo compuesta por 385 personas de al menos 16 años de edad de la población general. El análisis de consistencia interna de la escala mostró una alta confiabilidad, y el análisis factorial exploratorio una estructura de tres factores de primer orden y un factor general de segundo orden que explicaron $44.7 \%$ de la varianza. El análisis factorial confirmatorio comprobó esta estructura y arrojó buenos índices de ajuste relativo y general para la escala. En conclusión, el instrumento que aquí se presenta es confiable para evaluar la compasión en la población mexicana.
\end{abstract}

Palabras clave: Compasión; Sufrimiento; Reacción afectiva; Alivio del sufrimiento.

\begin{abstract}
Compassion, as a construct used in the present study, was defined as the deep desire to alleviate one's own suffering and that of others. The present study describes the process of construction of a compassion scale for Mexican population, as well as the evaluation of its psychometric properties. The sample included 385 participants from the general population, who were at least 16 years old. The analysis of the internal consistency of the scale showed high reliability; the exploratory factor analysis obtained a structure of three factors of first order, and a general factor of second order explaining $44.7 \%$ of the variance. The confirmatory factor analysis proved this structure and showed good indexes of both relative and general adjustment of the scale. In conclusion, this instrument is a reliable tool to assess compassion in the Mexican population.
\end{abstract}

Key words: Compassion; Suffering; Affective reaction; Alleving suffering.

\section{INTRODUCCIÓN}

C

ompasión es un concepto que ha suscitado controversias relacionadas con su definición debido a que se ha intentado explicar desde diferentes perspectivas teóricas y filosóficas con resultados que en ocasiones han sido incluso contradictorios (Goetz, Keltner y Simon-Thomas, 2010). Al margen de dichas controversias, la psicología basada en evidencias ha retomado el concepto pretendiendo una definición operacional que permita su estudio sistemático, y ha proporcionado puntos de referencia confiables para la comprensión del fenómeno (Goetz et al., 2010; Singer y Steinbeis, 2009).

Una de las definiciones más utilizadas en el estudio de la compasión señala que es una profunda conciencia del sufrimiento de otro y el subsecuente deseo de aliviarlo (Bornemann y Singer, 2013; Goetz et al., 2010; Gyatso, 2001; Ozawa, Dodson, Raison y Negi, 2012; Singer y Kilmecki, 2014).

\footnotetext{
${ }^{1}$ Se agradece a la Comisión Nacional de Ciencia y Tecnología (CONACyT), ya que el presente trabajo fue posible gracias a la beca no. 449437 otorgada al primer autor del presente artículo con no. de becario 299644 durante sus estudios doctorales.

${ }^{2}$ Facultad de Psicología, Universidad Nacional Autónoma de México, Av. Universidad 3004, Col. Copilco Universidad, Del. Coyoacán, Ciudad de México, México, correos electrónicos. moca99_99@yahoo.com y alejandrolopez2024@gmail.com. Artículo recibido el 22 de enero y aceptado el 20 de febrero de 2018.
} 
Singer y Steinbeis (2009) apuntan que tal definición está relacionada con las tradiciones contemplativas orientales, las cuales frecuentemente se refieren a la compasión de dos maneras: como un estado emocional caracterizado por sentimientos de calidez, amor y preocupación por el bienestar de los demás, y como una motivación o aspiración de ayudar a otros y promover su bienestar.

El concepto de compasión ha estado siendo integrado consistentemente a las psicoterapias occidentales (Germer y Siegel, 2012), y aunque en un principio se señalaba su semejanza (y posible equivalencia) con conceptos como empatía (Phillips y Taylor, 2009), los avances en la investigación en psicología y las neurociencias han mostrado paulatinamente que la compasión es un fenómeno bien diferenciado de otros procesos que pudieran parecer similares, principalmente por la participación de estructuras cerebrales asociadas con el bienestar y los afectos positivos, así como por la existencia de una clara motivación para aliviar el sufrimiento, lo que se traduce en el aumento de conductas prosociales (Gilbert y Choden, 2013; Singer y Kilmecki 2014). Por consiguiente, es importante señalar que la compasión no debe confundirse con la empatía, pues esta última es solo un componente afectivo de la compasión (Mercadillo, 2012).

Desde una perspectiva evolutiva, se considera que la compasión evolucionó con el apego en los mamíferos y está arraigada en la capacidad de experimentar sentimientos afiliativos, de ser amado y ser capaz de amar a otros como elementos indispensables de un proceso adaptativo; la compasión, como una respuesta ante el sufrimiento de uno mismo y de los demás, se puede considerar un elemento que forma parte de una serie de repertorios conductuales que sirven para apoyar la supervivencia propia y del resto de los miembros de la especie (Gilbert, 2013).

La evidencia obtenida hasta ahora indica que la compasión tiene efectos benéficos en la salud, toda vez que ayuda a las personas a estar fisiológicamente mejor reguladas en relación a las hormonas de estrés, el sistema inmunológico, el procesamiento cortical frontal y la creatividad, al tiempo que fomenta estados de bienestar y ayuda a las personas a hacer frente de mejor manera a toda una variedad de deseos, fantasías, miedos, enojos, re- cuerdos traumáticos y situaciones de estrés (Gilbert, 2013; Leaviss y Uttley, 2015; Ozawa et al., 2012).

Las intervenciones psicológicas centradas en la compasión se han analizado de manera sistemática en el ámbito aplicado, y diversos estudios han utilizado estas intervenciones en poblaciones con trastornos como esquizofrenia (Braehler et al., 2013; Laithwaite et al., 2009; Mayhew y Gilbert, 2008), sintomatología ansiosa y depresiva (Gilbert y Irons, 2004; Gilbert y Procter, 2006), trastorno bipolar (Laithwaite et al. 2009), desórdenes crónicos de personalidad (Lucre y Corten, 2013), acné crónico (Kelly, Zuroff y Shapira, 2009), trastornos alimentarios (Gale, Gilbert, Read y Goss, 2014), efectos posteriores a traumas (Beaumont et al. 2012), lesiones cerebrales por accidentes de tráfico (Ashworth, Gracey y Gilbert, 2011), tabaquismo (Kelly, Zuroff, Foa y Gilbert, 2010), depresión, ansiedad, trastorno obsesivo-compulsivo, ansiedad social y autolesiones deliberadas (Judge, Cleghorn, McEwan y Gilbert, 2012).

La creciente incorporación de protocolos de entrenamiento en compasión en el ámbito psicoterapéutico tiene implicaciones importantes para los profesionales del área, pues se traduce en una creciente necesidad de disponer de métodos de medición confiables que permitan evaluar los efectos de las intervenciones utilizadas y, tal como señalan Martins, Nicholas, Shaheen, Jones y Norris (2013), identificar con claridad los niveles de compasión necesarios para producir cambios conductuales.

Actualmente, es posible encontrar en la literatura internacional tres escalas específicas que han medido la compasión en población universitaria (Chang, Fresco y Green, 2014; Martins et al., 2013; Sprecher y Fehr, 2005), así como instrumentos psicométricos que miden constructos asociados a la compasión, como la autocompasión (Neff, 2003), que se dirige al propio sufrimiento y el deseo de aliviarlo, en tanto que la compasión está orientada a aliviar el sufrimiento de los demás.

La literatura existente acerca de la compasión suele mostrar algunas variaciones en las dimensiones que conforman el constructo. En los estudios en que se han desarrollado escalas específicas de compasión, la estructura factorial del constructo ha sido diversa; por ejemplo, la Escala 
de Amor Compasivo por los Otros Cercanos y la Humanidad, de Sprecher y Fehr (2005), fue diseñada inicialmente para ser una escala de tres factores; sin embargo, se mostró como una escala de un solo factor que incluía reactivos que evalúan ternura ante el sufrimiento, comprensión, ayuda y sacrificio. Por su parte, Martins et al. (2013) obtuvieron en su escala una estructura de dos factores, el primero de los cuales indaga la cantidad de diversos tipos de apoyo o ayuda a los demás que proporcionan las personas (p.e. “¿Cuánto de su tiempo libre dedicaría a trabajar para un extraño que necesita sus habilidades pero no puede pagarlo?"), y el segundo la frecuencia con que se proporciona algún tipo de ayuda a otros o en cuántas ocasiones se dejan de emitir conductas que pudieran generar daño a otros (p.e. “¿Cuántas veces te negarías el placer de algo que causa dolor a otros?"). Por último, la escala desarrollada por Chang et al. (2014) posee asimismo una estructura de dos factores: el primero mide la empatía como una reacción afectiva ante la situación de vida de otros, y el segundo el deseo de aliviar el sufrimiento de otros.

Como se puede observar, la estructura factorial de las escalas existentes resulta variable; no obstante, dos de esas escalas coinciden en incorporar reactivos que evalúan la existencia de una reacción afectiva ante el sufrimiento (Chang et al., 2014; Sprecher y Fehr, 2005), y todas ellas coinciden en la necesidad de incorporar reactivos que evalúen la motivación para ayudar a otros o de aliviar su sufrimiento, particularmente la escala de Martins et al. (2013), que concentra la cantidad y frecuencia de formas de ayuda o apoyo muy específicas.

A pesar de la falta de coincidencia en cuanto a los factores en los citados estudios, los resultados obtenidos coinciden de manera general con lo señalado por Ozawa et al. (2012), quienes indican que, estrictamente hablando, los principales ingredientes o condiciones preestablecidas para que la compasión tenga lugar son al parecer la capacidad de reconocer el sufrimiento en los otros y experimentar una reacción afectiva ante ese sufrimiento, de modo que si ambos están presentes, el deseo de aliviar este sufrimiento es posible, pero si falta alguno, dicha compasión no se produce.
El estudio formal de la compasión en México en el ámbito de la psicoterapia es muy reciente; sin embargo, la posibilidad de que pueda producir resultados confiables depende en gran medida de la existencia de una escala psicométrica adecuada para la población mexicana.

Así, el objetivo de este estudio fue el desarrollo de dicha escala, al considerar que la relevancia cultural de un instrumento que indague la compasión resulta evidente; de igual modo, el tipo de población a partir de la cual se construyeron las escalas existentes, muchas de hechas destinadas a estudiantes universitarios, podría dificultar la evaluación del constructo en la población general de México, por lo cual el desarrollo desde cero de esta escala representa un beneficio para la investigación aplicada.

\section{MÉTODO}

\section{Participantes}

Participaron 385 hombres y mujeres de la población general. Cómo criterios de inclusión se consideraron la nacionalidad mexicana, una edad mínima de 16 años y la disposición explícita a participar en el estudio.

\section{Instrumento}

Para construir los reactivos se utilizaron diversos métodos; algunos de ellos se desarrollaron por medio de la revisión de la teoría existente acerca de la compasión, otros fueron construidos a partir de una lista de situaciones en las cuales pudiera manifestarse la compasión, lista la cual fue solicitada a cuatro profesionales estudiosos del tópico incorporados al programa de posgrado en Psicología de la Universidad Nacional Autónoma de México; finalmente, también se elaboraron reactivos por medio de un análisis de contenido realizado con las respuestas de cincuenta informantes de la población general.

A partir de este trabajo, se obtuvo un listado inicial de 133 reactivos distribuidos en tres dimensiones propuestas a partir de la revisión de la literatura: una dimensión cognitiva en la que se incorporaban reactivos encaminados a evaluar la 
capacidad de las personas para reconocer situaciones de sufrimiento en los demás; una segunda dimensión que contenía reactivos orientados a evaluar las reacciones afectivas de las personas ante situaciones de sufrimiento, y una más que analizaba el deseo de las personas de aliviar el sufrimiento. Después, se realizó un piloteo de esta primera versión del cuestionario con diez personas, luego del cual se eliminaron 37 reactivos ya que su redacción se consideró poco clara, ambigua, o porque resultaban redundantes con otros reactivos presentes en la misma dimensión.

Los 96 reactivos restantes se sometieron al análisis de tres expertos que evaluaron su importancia a partir de la definición teórica y las dimensiones propuestas, así como que se cubrieran las dimensiones de interés por los reactivos incluidos en cada una de ellas. A partir de esta revisión se eliminaron 20 reactivos más que no cumplieron con los criterios de validez establecidos, permaneciendo 76 reactivos que se incorporaron a una versión del cuestionario para su aplicación en una escala tipo Likert con siete opciones de respuesta, que iban de "nunca" a "siempre".

La escala fue autoaplicable, por lo cual se elaboró una versión de lápiz y papel que podía responderse directamente, así como un formulario digital en la plataforma Google Docs.

\section{Recolección de datos}

Durante la aplicación, la mitad de los participantes contestó el cuestionario de lápiz y papel, y la otra mitad el formulario digital; los participantes que respondieron este último recibían una invitación única por correo electrónico, la cual incluía un enlace al formulario para que respondieran el cuestionario en caso de que estuvieran de acuerdo en participar en el estudio. Los datos se codificaron y procesaron mediante el paquete SPSS, versión 21.

\section{RESULTADOS}

\section{Características de los participantes}

De los participantes, $70 \%$ fueron mujeres y $30 \%$ hombres; $24 \%$ contaba con el nivel básico de edu- cación y $76 \%$ con estudios profesionales; de igual modo, 69\% reportó tener una práctica espiritual (religión, filosofía, idea propia de espiritualidad o actividad espiritual), mientras que $31 \%$ restante respondió no tener esas prácticas.

Del total de personas que respondieron practicar alguna forma de espiritualidad, 64.7\% dijeron profesar el catolicismo, $11.2 \%$ el cristianismo, $8.9 \%$ alguna forma de budismo o alguna práctica que ellos relacionaban con dicha creencia, y 10.1\% manifestó llevar otro tipo de práctica que consideraba formas de espiritualismo, como el yoga, la meditación o una idea de espiritualidad propia.

La media de edad fue de 37.6 años, con una desviación estándar de 12.7. De esos participantes, 228 residían en la Ciudad de México, 65 en el Estado de México, 43 en el de Veracruz, 22 en el de Coahuila y los 28 restantes en diversos estados de la República Mexicana.

\section{Confiabilidad y validez}

Tras llevar a cabo un análisis de discriminación de reactivos por distribución de frecuencias, discriminación de reactivos por sesgo y curtosis y discriminación de reactivos por diferencias en grupos extremos, se eliminaron ocho reactivos por concentrar más de $50 \%$ de respuestas en una sola opción de la codificación; dos reactivos por poseer puntajes de curtosis mayores a 2 , y uno mediante una prueba $t$ para muestras independiente por no discriminar entre grupos extremos.

Con los 66 reactivos así obtenidos, se hizo un análisis de confiabilidad por medio de una prueba alfa de Chronbach, a partir de la cual se eliminó un reactivo más cuya correlación elemento-total corregida fue menor de .20 , y se solicitó la medida de adecuación muestral de Kaiser-Meyer-Olkin (KMO) y la prueba de esfericidad de Bartlett. El valor de la prueba KMO fue .943 , lo que lleva a suponer que el tamaño de la muestra tenía suficiente adecuación, y el de la prueba de esfericidad de Bartlett fue igual a $10940.824(\mathrm{df}=861$, $p<0.001$ ). Estos resultados confirmaron que era factible realizar un análisis factorial exploratorio con los datos obtenidos.

El primer análisis factorial exploratorio con el método de extracción de máxima verosimilitud y una rotación varimax permitió eliminar 31 reac- 
tivos que no registraron cargas mayores a .40, o que no se agruparon adecuadamente dentro de un factor con al menos tres reactivos; luego, los 34 reactivos restantes se sometieron nuevamente al análisis de confiablidad y al análisis factorial exploratorio, de los que se obtuvo una estructura de tres factores: Motivación para aliviar el sufrimiento, Reacción afectiva ante el sufrimiento y Compasión hacia los animales.

Después, mediante la utilización del programa AMOS 24, se realizó un análisis factorial confirmatorio con base en el programa de ecuaciones estructurales (Byrne, 2001; Kline, 2005). A partir de dicho análisis se eliminaron aquellos reactivos que no tuvieron una carga mayor a .40. De igual modo, se analizó la matriz de covarianzas residuales estandarizadas con la finalidad de mejorar el ajuste del modelo eliminando aquellos reactivos cuya covarianza con otros reactivos fuera alta $(\leq 2)$. Al final de este proceso se eliminaron 17 reactivos más.

Con los 17 reactivos restantes se repitieron el análisis alfa de Cronbach y el factorial exploratorio. El coeficiente alfa total resultante fue de .90; de igual modo, la confiabilidad de los tres factores resultantes fue alta, y la varianza total explicada de $53.59 \%$. Dichos coeficientes, así como los reactivos de cada factor, se muestran en la Tabla 1.

Tabla 1. Subescalas, cargas factoriales obtenidas para los reactivos y varianza explicada de la Escala de Compasión para Población Mexicana.

\begin{tabular}{|c|c|c|c|}
\hline \multirow[b]{2}{*}{ REACTIVOS } & \multicolumn{3}{|c|}{ Factores } \\
\hline & $\begin{array}{c}1 \\
\text { Motivación } \\
\text { para aliviar } \\
\text { el sufrimiento }\end{array}$ & $\begin{array}{c}2 \\
\text { Reacción } \\
\text { afectiva ante } \\
\text { el sufrimiento }\end{array}$ & $\begin{array}{c}3 \\
\text { Compasión } \\
\text { hacia } \\
\text { los animales }\end{array}$ \\
\hline $\begin{array}{l}\text { 70. Cuando veo que alguien está pasando un momento difícil, le pregunto } \\
\text { si puedo ayudarlo. }\end{array}$ & .744 & & \\
\hline $\begin{array}{l}\text { 64. Cuando alguien está sufriendo un duelo soy el primero en intervenir } \\
\text { y ayudar. }\end{array}$ & .670 & & \\
\hline 66. Cuando otros sienten tristeza, trato de confortarlos. & .668 & & \\
\hline $\begin{array}{l}\text { 20. Cuando veo que una persona se siente sola, siento deseos de ofrecerle } \\
\text { mi compañía. }\end{array}$ & .632 & & \\
\hline 36. Si veo que alguien necesita un consejo, trato de decirle algo que le ayude. & .578 & & \\
\hline 60. Dedico parte de mi tiempo libre a ayudar a los demás. & .530 & & \\
\hline $\begin{array}{l}\text { 2. Si alguien necesita ayuda económica, se la proporciono si tengo } \\
\text { la posibilidad. }\end{array}$ & .506 & & \\
\hline 55. Siento gran tristeza cuando veo a personas sin hogar. & & .800 & \\
\hline 65. Me duele la pobreza en el mundo. & & .796 & \\
\hline 48. Me entristece el sufrimiento de los seres humanos. & & .731 & \\
\hline 24. Siento gran pena por las personas que no tienen que comer. & & .662 & \\
\hline
\end{tabular}


Al analizar la correlación entre factores (Tabla 2), fue posible observar que existía una correlación significativa entre los factores $(p<.01)$, por lo que se llevó a cabo un análisis factorial de segundo orden mediante el método de extracción de factorización de ejes principales, a través del cual se comprobó la existencia de un factor de orden superior que explicó $44.7 \%$ de la varianza.

Tabla 2. Índices de correlación entre los factores de la Escala de Compasión.

\begin{tabular}{|l|c|c|c|}
\hline \multicolumn{1}{|c|}{ Subescala } & $\begin{array}{c}\text { Motivación para aliviar } \\
\text { el sufrimiento }\end{array}$ & $\begin{array}{c}\text { Reacción afectiva } \\
\text { ante el sufrimiento }\end{array}$ & $\begin{array}{c}\text { Compasión } \\
\text { hacia } \\
\text { los animales }\end{array}$ \\
\hline Deseo de aliviar el sufrimiento & 1.000 & & \\
\hline Reacción afectiva ante el sufrimiento & $.527^{* *}$ & 1.000 & 1.000 \\
\hline Compasión hacia los animales & $396^{* *}$ & $384 * *$ & \\
\hline
\end{tabular}

$* *$ Significativos $(p<.01)$

Hecho lo anterior, se realizó un análisis factorial confirmatorio de segundo orden con el propósito de examinar empíricamente la estructura factorial de la escala, fijándose en 1 las varianzas de las variables latentes.

Las varianzas de los términos de error se especificaron como parámetros libres. Se empleó el método de estimación de máxima verosimilitud. Los indicadores de bondad de ajuste absoluto obtenidos para la escala fueron, a saber: 1) $\chi^{2}$ entre los grados de libertad (el cociente debería ser $<4.00$, pues a menor índice, mejor ajuste) $=$ $236.837 / 115=2.05 ; 2)$ índice de ajuste comparativo de Bentler (CFI [Comparative fit index]). El valor debería acercarse a 0.90 , pues cuanto mayor sea el valor, mejor ajuste) $=0.962$, y 3 ) raíz cuadrada media del error de aproximación (RMSEA [Root mean square error of approximation]). Para un buen ajuste el valor debería ser $\leq 0.05)=0.05$. Los indicadores obtenidos de ajuste relativo, los cuales oscilaron entre 0 y $1(\leq .90$ generalmente se considera un buen ajuste), fueron los siguientes: 1) índice de ajuste normalizado (NFI [Normed fit index]) $=.92 ; 2$ ) índice de ajuste incremental (IFI [Incremental fit index]) $=.96 ; 3$ ) índice de ajuste comparativo [CFI, Comparative fit index]) $=.96$. En suma, los indicadores de bondad de ajuste para la Escala de Compasión para población mexicana mostraron niveles adecuados de ajuste a los datos, lo que confirma la estructura factorial obtenida en el análisis exploratorio.

En la Tabla 3 se expresan la media y desviación estándar de las puntuaciones de compasión por cada factor, así como la media total de la escala para toda la población participante en el estudio.

Tabla 3. Medias y desviaciones estándar de las subescalas de la Escala de Compasión.

\begin{tabular}{|l|c|c|}
\hline \multicolumn{1}{|c|}{ Subescala } & Media* & D. E. \\
\hline Motivación para aliviar el sufrimiento & 4.99 & 0.87 \\
\hline Reacción afectiva ante el sufrimiento & 5.74 & 1.13 \\
\hline Compasión hacia los animales & 5.51 & 1.19 \\
\hline Compasión total & 5.42 & 0.84 \\
\hline
\end{tabular}

\section{DISCUSIÓN}

La escala aquí presentada se diseñó a partir de una estructura de tres factores (cognitivo, afectivo y motivacional); sin embargo, de manera muy similar a lo hallado por Sprecher y Fehr (2005), la estructura factorial final de la escala se modificó ligeramente, conservando sin embargo el número de tres factores de primer orden (Deseo de aliviar el sufrimiento, Reacción afectiva ante el sufrimiento y Compasión hacia los animales), al que se agregó un factor de segundo orden llamado Compasión total.

En general, los factores obtenidos en la presente escala coincidieron conceptualmente con lo hallado en estudios como el de Sprecher y Fehr (2005) y Chang et al. (2014), quienes obtuvieron factores en los que se incorporaban reactivos que 
evalúan la reacción afectiva hacia el sufrimiento de otros, así como la motivación de disminuir el sufrimiento.

Otro aspecto importante del presente estudio es el modo consistente en que la motivación de aliviar el sufrimiento (aquí encontrada en el factor 1 por medio de reactivos que evalúan el acercamiento a los otros, la intención y la conducta orientada a aliviar el sufrimiento) es el elemento con mayor presencia y peso en la medición de la compasión, lo que concuerda con lo señalado por autores como Gilbert y Choden (2013) y Singer y Kilmecki (2014), que subrayan que este es un elemento que distingue la compasión de procesos que pudieran parecer semejantes, como la empatía.

Si bien durante el desarrollo de la escala se decidió incluir un factor cognitivo, mismo que indagaba el reconocimiento del sufrimiento en otros a partir de la sugerencia de Ozawa et al. (2012), quienes apuntan que el reconocimiento del sufrimiento en otros es un elemento sin el cual la compasión no se produce, tal proceso cognitivo parece estar implícito en la reacción afectiva ante el sufrimiento, y el motivo que podría explicar su ausencia en la estructura factorial final de la escala podría ser que dicho proceso ocurre frecuentemente fuera del control consciente de las personas que entran en contacto con el sufrimiento de otros seres vivos, reconociéndolo únicamente hasta que se produce una reacción afectiva.

Un elemento que resulta distinto en la presente escala al compararla con otras es la incorporación de reactivos que evalúan la compasión hacia los animales y que se agruparon en un solo factor. En términos teóricos, este es un resultado interesante ya que sugiere que la compasión no es un fenómeno que se limite a la interacción que el ser humano tiene con miembros de su misma especie (nivel en el que se han desarrollado las otras escalas existentes), sino que se extiende a otros seres vivos, en la medida en que reconoce en ellos la capacidad de experimentar sufrimiento y que posee la motivación para aliviarlo.

En conclusión, los resultados obtenidos en la presente investigación indican que el instrumento de compasión desarrollado es una medida valida y confiable de este constructo en la población mexicana.

Sobresale su similitud con la estructura factorial reportada en otros estudios, en los cuales es constante un factor que involucra el acercamiento a los otros, la intención de ayudarlos y la emisión de conductas muy específicas orientadas al alivio del sufrimiento, así como de un factor que evalúa reacciones emocionales ante el sufrimiento; la contribución del presente estudio es que a los dos factores mencionados se agrega por primera vez un factor que evalúa la compasión hacia los animales.

Entre las limitaciones del presente estudio se encuentran la imposibilidad parcial de generalizar los resultados a poblaciones distintas a la estudiada aquí, por lo cual su aplicación en grupos con distintas características es un área de oportunidad importante para el desarrollo de futuras investigaciones.

\section{REFERENCIAS}

Ashworth, F., Gracey, F. y Gilbert, P. (2011). Compassion focused therapy after traumatic brain injury: theoretical foundations and a case illustration. Brain Impairment. 12, 128-139. Recuperado de https://doi.org/10.1375/brim.12.2.128.

Bonermann, B. y Singer T. (2013). A cognitive neuroscience perspective. The resource model of compassion. En T. Singer y M. Bolz (Eds.): Compassion. Bridging practice and science (pp. 179-191). Munich: Max Planck Society.

Braehler, C., Gumley, A., Harper, J., Wallace, S., Norrie, J. y Gilbert, P. (2013). Exploring change processes in compassion focused therapy in psychosis: Results of a feasibility randomized controlled trial. British Journal of Clinical Psychology, 52, 199-214. Recuperado de http://onlinelibrary.wiley.com/doi/10.1111/bjc.12009/abstract.

Chang J., Fresco, J. y Green, B. (2014). The development and validation of the Compassion of Others' Lives Scale (The COOL Scale). International Journal of Humanities and Social Science, 4(5), 33-42. Recuperado de http://www.ijhssnet.com/ journals/Vol_4_No_5_March_2014/4.pdf.

Gale, C., Gilbert, P., Read, N. y Goss, K. (2014). An evaluation of the impact of introducing compassion focused therapy to a standard treatment programme for people with eating disorders. Clinical Psychology and Psychotherapy, 21, 1-12. Recuperado de https://www.ncbi.nlm.nih.gov/pubmed/22740105. 
Germer, C. y Siegel, R. (2012). Wisdom and compassion: two wings of a bird. En R. Siegel y C. Germer (Eds.): Wisdom and compassion in psychotherapy: Deepening mindfulness in clinical practice (pp. 7-34). New York: The Guilford Press.

Gilbert, P. (2013). The flow of life. An evolutionary model of compassion. En T. Singer y M. Bolz (Eds.): Compassion. Bridging practice and science (pp.127-149). Munich: Max Planck Society.

Gilbert, P. y Choden (2013). Mindful compassion. London, UK: Constable-Robinson.

Gilbert, P. e Irons, C. (2004). A pilot exploration of the use of compassionate images in a group of self-critical people. Memory, 12, 507-516. Recuperado de https://www.ncbi.nlm.nih.gov/pubmed/15487546.

Gilbert, P. y Procter, S. (2006). Compassionate mind training for people with high shame and self-criticism: Overview and pilot study of a group therapy approach. Clinical Psychology and Psychotherapy, 13, 353-379.

Goetz, J.L., Keltner, D. y Simon-Thomas, E. (2010). Compassion: An evolutionary analysis and empirical review. Psychological Bulletin, 136(3), 351-374. Recuperado de http://doi.org/10.1037/a0018807.

Gyatso, T. (2001). An open heart: Practising compassion in every day life. New York: Little, Brown and Company.

Judge, L., Cleghorn, A., McEwan, K. y Gilbert, P. (2012). An exploration of group-based compassion focused therapy for a heterogeneous range of clients presenting to a community mental health team. International Journal of Cognitive Therapy, 5, 420-429. Recuperado de https://www.ncbi.nlm.nih.gov/pubmed/24217864.

Kelly, A.C., Zuroff, D.C., Foa, C.L. y Gilbert, P. (2010). Who benefits from training in self-compassionate self-regulation? A study of smoking reduction. Journal of Social and Clinical Psychology, 29, 727-755. Recuperado de http://guilfordjournals.com/doi/abs/10.1521/jscp.2010.29.7.727.

Kelly, A.C., Zuroff, D.C. y Shapira, L.B. (2009). Soothing oneself and resisting self-attacks: the treatment of two intrapersonal deficits in depression vulnerability. Cognitive Therapy and Research, 33, 301-313. Recuperado de https://link.springer. com/article/10.1007/s10608-008-9202-1.

Laithwaite, H., O'Hanlon, M., Collins, P., Doyle, P., Abraham, L., Porter, S. y Gumley, A. (2009). Recovery after psychosis (RAP): A compassion focused programme for individuals residing in high security settings. Behavioural and Cognitive Psychotherapy, 37, 511-526. Recuperado de https://www.ncbi.nlm.nih.gov/pubmed/19765353.

Leaviss, J. y Uttley, L. (2015). Psychotherapeutic benefits of compassion-focused therapy: An early systematic review. Psychological Medicine, 45(5), 927-945. Recuperado de http://doi.org/10.1017/S0033291714002141.

Lucre, K. y Corten, N. (2013). An exploration of group compassion-focused therapy for personality disorder. Psychology and Psychotherapy, 86, 387-400. Recuperado de https://www.ncbi.nlm.nih.gov/pubmed/24217864.

Martins, D., Nicholas, N.A., Shaheen, M., Jones, L. y Norris, K. (2013). The development and evaluation of a compassion scale. Journal of Health Care for the Poor and Underserved, 24(3), 1235-1246. Recuperado de http://doi.org/10.1353/ hpu.2013.0148.

Mayhew, S. y Gilbert, P. (2008). Compassionate mind training with people who hear malevolent voices: a case series report. Clinical Psychology and Psychotherapy, 15, 113-138. Recuperado de https://www.ncbi.nlm.nih.gov/pubmed/19115433.

Mercadillo, R. (2012). Retratos del cerebro compasivo: una reflexión en la neurociencia social, los policías y el género. México: Centro de Estudios Filosóficos, Políticos y Sociales Vicente Lombardo Toledano.

Neff, K.D. (2003). The development and validation of a scale to measure self-compassion. Self and Identity, 2(3), 223-250. Recuperado de http://psycnet.apa.org/record/2003-05728-004.

Ozawa S., B., Dodson L., B., Raison, C. y Negi, L. (2012). Compassion and ethics: Scientific and practical approaches to the cultivation of compassion as a foundation for ethical subjectivity and well-being. Journal of Healthcare, Science and the Humanities, 2(1), 145-161.

Phillips, A. y Taylor, B. (2009). On kindness. London: Hamish Hamilton.

Singer, T. y Kilmecki, O.M. (2014). Empathy and compassion. Current Biology, 24(18), 875-878. Recuperado de http://www.cell. com/current-biology /abstract/S0960-9822(14)00770-2.

Singer, T. y Steinbeis, N. (2009). Differential roles of fairness-and compassion-based motivations for cooperation, defection, and punishment. Annals of the New York Academy of Sciences, 1167, 41-50. Recuperado de http://doi:10.1111/j.17496632.2009.04733.x.

Sprecher, S. y Fehr, B. (2005). Compassionate love for close others and humanity. Journal of Social and Personal Relationships, 22, 629-651. Recuperado de http://journals.sagepub.com/doi/abs/10.1177/0265407505056439. 